\title{
Peer Reviewers for the Journal of the American Board of Family Medicine in 2012
}

Phillip Lupo, Jr., MLIS, Anne Victoria Neale, PhD, MPH, and Marjorie A. Bowman, MD, MPA

We thank our peer reviewers for their time, expertise, and dedication to the Fournal of the American Board of Family Medicine ( $A A B F M)$. The $7 A B F M$ peer reviewers serve a vital role in contributing to the field of family medicine and the scientific community. We would like to take this opportunity to acknowledge and thank our peer reviewers for their support during the last year. In 2012, 372 individuals provided 476 peer reviews and advice regarding the suitability of articles for publication in the $7 A B F M$. Reviewers took an average of 22 days to submit a review.

\section{Top Reviewers}

We would like to recognize our top reviewers. Each of the manuscripts submitted to the $7 A B F M$

Conflict of interest: The authors are editors and staff of the 7ABFM. that undergo peer review is evaluated and scored by the editors; in the list below, peer reviewers with an asterisk next to their name are in the top $12 \%$ (based on evaluation scores plus quantity of reviews) of all reviewers in 2012.

\section{Seeking New Reviewers!}

We also enjoy welcoming new peer reviewers into the fold. Peer reviewing is a vital service activity that helps authors and researchers improve their work. Reviewers are always free to decline a request or to indicate periods of unavailability. If you would like to volunteer to review for the $7 A B F M$, please download and complete the Peer Reviewer Form, which can be accessed at http://jabfm.org/site/ misc/PeerReviewForm.doc, and E-mail it along with your curriculum vitae to jabfm@med.wayne. edu or fax it to 313-577-9828. Thank you!

Ya'aqov M. Abrams
John Abramson
David A. Acosta
David C. Agerter
Grace A. Alfonsi
William A. Alto*
Gail M. Amundson
Mark D. Andrews
Christine Arenson
Nancy T. Artinian
John Bachman
Elisabeth L. Backer
Michael T. Baer
Suzanne Bakken
Laura-Mae Baldwin
Mikhail Bargan
Steven Barnett
Donald Barr
Laurence Bauer
Max Bayard

Elizabeth A. Bayliss
John W. Beasley
Ian M. Bennett*
Terrell Benold
Alfred O. Berg*
Klea D. Bertakis
Frances E. Biagioli
Janet Bickel
Juliann Binienda*
Rajshekhar Bipeta
Barry Bleidt
Alan Blum
Debra Boardley*
George W. Bock
John M. Boltri*
Jason C. Booza
Pranjal K. Boruah
Kent Bream
Erica Brode
Howard Brody*

Elizabeth A. Bayliss

John W. Beasley

Ian M. Bennett*

Terrell Benold

Alfred O. Berg*

Klea D. Bertakis

Frances E. Biagioli

Janet Bickel

Rajshekhar Bipeta

Barry Bleidt

Alan Blum

Debra Boardley*

George W. Bock

John M. Boltri*

Jason C. Booza

Pranjal K. Boruah

Erica Brode
Fredrik F. Broekhuizen

Carlos Brotons

Richard L. Brown

Steven R. Brown

Sean T. Bryan

Subbaraju Budharaju

Sandra K. Burge

Peter J. Carek

Andrew J. Cave

William E. Cayley, Jr.

Sherman Chamberlain

Sabrina M. Chase

Ranee Chatterjee

Stephen Y. Chen

Robin E. Clark

Richard D. Clover

Allison M. Cole

Monica K. Crane

Paul F. Crawford III

Jesse C. Crosson* 
Michael A. Crouch

Brian K. Crownover

Richard W. Crummer

Larry Culpepper*

Doyle M. Cummings

Timothy P. Daaleman

Jeanette M. Daly

Peter G. Danis III

Robert J. Darios

Alan K. David

Melinda M. Davis

Peter A. de Schweinitz

Lauren DeAlleaume

M. Dianne Delva

Jennifer E. DeVoe*

L. Miriam Dickinson*

Joseph R. DiFranza

Lisabeth F. DiLalla

Paul P. Doghramji

William J. Doherty

Tim Doran

Steven A. Dosh

Tony Dowell

S. Shevaun Duiker

Gareth Dutton

Milton Eder

Marlene J. Egger

Nancy C. Elder

William G. Elder, Jr.

Kristin K. Elliott

John W. Ely*

Shannon K. English

James A. Espinosa

Lyle J. Fagnan

Gail Fahoome

Tim D. Feit

Joshua J. Fenton*

Douglas Fernald

Jeanne M. Ferrante*

Daron G. Ferris

Edgar Figueroa

Robert L. Findling

Kenneth S. Fink*

Kevin Fiscella

Alan B. Fleischer, Jr.

Colleen T. Fogarty

Arthur Fournier

Peter Franks

Callum G. Fraser

Linda M. French

John J. Frey III

Harsha V. Ganga

Elizabeth Garrett
Suzanne Gehl

Christa George

Mark D. Gideonsen*

Valerie J. Gilchrist

James M. Gill

Mary Lou Gillard

Dwenda K. Gjerdingen

Adam O. Goldstein*

Rise B. Goldstein

Bharat Gopal

Beverly B. Green*

Larry A. Green*

Jessica L. Greenwood

Tomas L. Griebling

Kim Griswold*

Joseph G. Grzywacz

Alexander B. Guirguis

Robert Gwyther

David L. Hahn

Ricardo Hahn

Scott Haldeman

Irene Hamrick

Cynthia Haq

Diane M. Harper

Ila M. Harris

Susan Hart-Hester

Robert L. Hatch

Joel J. Heidelbaugh

Robin Helm

Margaret Helton

Nikhil Hemady

Eric Henley

Daniel Hinthorn

Richard Hoffman

Don Holbert

Phyllis Horns

Michael A. Horst

Susan Hsieh

Lauren S. Hughes

Katherine Hurst

Jihad Irani

Jon Eric Isaacson

Henry R. Ivey, Jr.

Carlos Roberto Jaen

Paul A. James

James Janisse

Wiley D. Jenkins

Anthony F. Jerant

Masahito Jimbo*

Mark S. Johnson

Samuel M. Jones

Linda S. Kahn*

Nitin Kashyap david katerndahl

Omar A. Khan

Niharika Khanna

Morteza Khodaee

Sanford R. Kimmel

Kenneth Kirkwood

Lyndee Knox

Richelle J. Koopman

Alex H. Krist

Jerry E. Kruse

Robin L. Kruse

Grace M. Kuo

Anton J. Kuzel

Robert Langan

Carol J. Lange

Jason S. Lanham

Frank Lawler

Aaron M. Lear

Kenyatta Lee

Shoou-Yih D. Lee

Patricia M. Lenahan

Sarah Lesko*

James E. Lessenger

Lenard I. Lesser*

Lawrence Leung*

Barcey T. Levy

Winston Liaw*

Deerajnath Lingutla

Everett E. Logue

Carmela G. Lomonaco

David P. Losh

Jorgen Lous

Margaret M. Love

Todd Lucas

Ann Macaulay

Michael V. Maciosek

Laura Makaroff*

William T. Manard

Tsveti Markova*

John Marley

Cara Marshall

Kate Martin

Samuel C. Matheny

Oksana Matvienko

Larry B. Mauksch

Charles Maynard

Catherine McCarthy

John McCarthy

Emily K. McCoy

Laura McCray

Michael M. McKee

James F. McKenzie

J L. Michener 
Sandra Miller

Fred Miser

James W. Mold*

Christopher P. Morley

Anne L. Mounsey

Karen Muchowski

Elie Mulhem

Marlon P. Mundt

Joseph M. Murley

John B. Murphy

Zsolt J. Nagykaldi*

David B. Nash

Jon O. Neher

Lynne S. Nemeth

Robert J. Newman

Warren P. Newton

Giang T. Nguyen

Thomas E. Norris*

Mary Patricia Nowalk

Alane B. O'Connor

David T. O'Gurek

Willie H. Oglesby

M N. Oliver*

Hean T. Ong

Steven M. Ornstein

Nancy Pandhi

Veena Panthangi

Donald Pathman*

Valory N. Pavlik

Eribeth K. Penaranda

Darcy F. Phelan

Robert L. Phillips, Jr.

Joesph M. Pierre

Charles D. Ponte

Robert E. Post

Michael B. Potter*

Shailendra Prasad

Michael D. Prislin

Perry A. Pugno*

Howard K. Rabinowitz

Marsha A. Raebel

Kalyanakrishnan Ramakrishnan

Ravishankar Ramaswamy

Brian V. Reamy

Richard L. Reed

Joanne L. Reeve

Michael D. Reis

Robert L. Rhyne

Thomas C. Ricketts

Hector P. Rodriguez

Michelle A. Roett
John C. Rogers

James E. Rohrer

Margaret S. Rosenberg

Michael P. Rosenthal

Thomas C. Rosenthal

Robert G. Ross

Linda Roth

Robert S. Rudin

George Rust

Fred Saad

Enrique Sanchez Valdivieso

James Sanders

Sudhakar Sathyanarayanan

David Satin

Judith A. Savageau

Martin Schencking

Joseph E. Scherger

Rebecca Schnall

Sarina Schrager*

Harvey Schwartz

Thomas L. Schwenk

Christopher N. Sciamanna

H R. Searight

Dean A. Seehusen*

Timothy H. Self

Egambaram Senthilvel

Patricia S. Sexton

Sachil Shah

Javad Shahidi

Eric K. Shaw*

Saima Siddiqui

William J. Sieber

Victor S. Sierpina

Irit Sinai

Peter C. Smith

Leif I. Solberg

John G. Spangler

William Spears

Stuart M. Speedie

John B. Standridge

Joseph B. Stanford

Ra Nae Stanton

Elizabeth W. Staton

Jeffrey A. Stearns

CDR Mark B. Stephens

Gayle G. Stephens

Nancy G. Stevens

Gregg D. Stoner

John E. Sutherland

Christine Sutine Chang

Paul R. Swank
Daniel J. Tancredi

Peter G. Teichman

Jonathan L. Temte*

Chris M. Terpening

Joshua Tessier

Clayton L. Thomason

Dixie L. Thompson

Jon S. Thompson

Tom Tomlinson

John M. Tovar

Hung Fu Tseng

Joanne Turnbull

Carole C. Upshur

Alan C. Utter

Anthony F. Valdini

Liset van Dijk

Daniel J. Van Durme

Mohamud A. Verjee

Anthony J. Viera

Daniel C. Vinson

Gordon Walbroehl

Eric Wall*

Alison M. Walton

Molly E. Waring

Marilyn T. Wayland

Barry D. Weiss

Brian J. Wells

Frances K. Wen

James J. Werner

David R. West

John M. Westfall*

Randy K. Wexler

Brett White

Elizabeth A. Wiley*

Thad Wilkins

Joanne E. Wilkinson

Robert L. Williams

Summer Williams

Paul S. Williamson

Robin Winter

Leslie E. Wolf

L. Thomas Wolff

W. Michael Woods

Imam M. Xierali*

Jinping $\mathrm{Xu}$

Mark J. Yaffe

Barbara P. Yawn

Atif Zafar

Philip Zazove

Therese Zink

Adam J. Zolotor

*Top reviewers in 2012 (based on quantity and quality of peer reviews written) 